\title{
MULTIDIMENSIONAL HYBRID BOUNDARY VALUE PROBLEM
}

\author{
Marzena Szajewska ${ }^{a, *}$, Agnieszka TereszkiewiCz $^{b}$ \\ ${ }^{a}$ Institute of Mathematics, University of Bialystok, $1 M$ Ciolkowskiego, PL-15-245 Bialystok, Poland \\ ${ }^{b}$ Bialystok University of Technology, Faculty of Civil and Environmental Engineering, 45 E Wiejska, PL-15-351 \\ Bialystok, Poland \\ * corresponding author: m.szajewska@math.uwb.edu.pl
}

Abstract. The purpose of this paper is to discuss three types of boundary conditions for few families of special functions orthogonal on the fundamental region. Boundary value problems are considered on a simplex $F$ in the real Euclidean space $\mathbb{R}^{n}$ of dimension $n>2$.

KEYworDs: hybrid functions, Dirichlet boundary value problem, Neumann boundary value problem, mixed boundary value problem.

\section{INTRODUCTION}

The boundary value problems, considered in the paper, is a generalization of [24] in which the authors presented two-dimensional hybrids with mixed boundary value problems. Here we take a real Euclidean space $\mathbb{R}^{n}$ of dimension $n$ on finite regions $F \subset \mathbb{R}^{n}$ that are polyhedral domains. The aim of this paper is to seek solutions of the Helmholtz equation with mixed boundary condition by analogy to two-dimensional cases. The solutions are presented as expansions into a series of special functions that satisfy required conditions at the $(n-1)$-dimensional boundaries of $F$. The recent discovery of special functions [5, 10, 11, 16, 19, 23. makes realization of this idea easy and straightforward in any dimension. The new functions, called 'multidimensional hybrids', satisfy the Dirichlet boundary condition on some parts of the boundary $F$ and Neumann on the remaining ones. The methods used in the paper are the standard methods of separation of variables for differential equations (see for example [15, 18]) and the branching rule method for orbits of reflection groups (see for example [19, 24, 28]). The boundary value conditions play an important role in mathematics and physics. They are used, for example, in the theory of elasticity, electrostatics and fluid mechanics [4, 9, 26].

In \$2 we present the well known Helmholtz equation and three types of boundary conditions. In $\S 3$, we recall some facts about finite reflection groups. The next Section is devoted to special functions, projection matrices and branching rules. In $\$ 5$ we present $3 \mathrm{D}$ cases in details, namely $B_{3}, C_{3}, C_{2} \times A_{1}, G_{2} \times A_{1}, A_{1} \times$ $A_{1} \times A_{1}$. In the Appendix we list tables containing the values of functions on the boundaries of fundamental region.

\section{HeLmHoltz EQUATION AND BOUNDARY CONDITIONS}

In this paper we consider the partial differential equation called the homogeneous Helmholtz equa- tion [15, 18, 25, and references therein]:

$$
\Delta \Psi(\mathbf{x})=-\mathrm{w}^{2} \Psi(\mathbf{x}),
$$

where w-positive real constant, $\mathbf{x}=\left(y_{1}, \ldots, y_{n}\right)$ is given in Cartesian coordinates and $\Delta=\sum_{i=1}^{n} \frac{\partial^{2}}{\partial y_{i}^{2}}$.

Using a standard method of separation of variables for (11) (see for example [15]) and searching for the solutions in the form $\Psi(\mathbf{x})=X_{1}\left(y_{1}\right) \cdots X_{n}\left(y_{n}\right)$, we have the following differential equation

$$
\begin{aligned}
& X_{1}^{\prime \prime} X_{2} \cdots X_{n}+X_{1} X_{2}^{\prime \prime} \cdots X_{n} \\
& \quad+\cdots+X_{1} X_{2} \cdots X_{n}^{\prime \prime}+\mathrm{w}^{2} X_{1} X_{2} \cdots X_{n}=0 .
\end{aligned}
$$

By introducing $-\mathrm{k}_{1}^{2}, \ldots,-\mathrm{k}_{n}^{2}$ so-called separation constants, we get the solution of 2 in the form

$$
\begin{aligned}
X_{1}^{1}\left(y_{1}\right) & =\cos \left(\mathrm{k}_{1} y_{1}\right), \\
& \vdots \\
X_{n-1}^{1}\left(y_{n-1}\right) & =\cos \left(\mathrm{k}_{n-1} y_{n-1}\right), \\
X_{n}^{1}\left(y_{n}\right) & =\cos \left(\mathrm{k}_{n} y_{n}\right), \\
X_{1}^{2}\left(y_{1}\right) & =\sin \left(\mathrm{k}_{1} y_{1}\right), \\
& \vdots \\
X_{n-1}^{2}\left(y_{n-1}\right) & =\sin \left(\mathrm{k}_{n-1} y_{n-1}\right), \\
X_{n}^{2}\left(y_{n}\right) & =\sin \left(\mathrm{k}_{n} y_{n}\right),
\end{aligned}
$$

where $k_{n}:=\sqrt{\mathrm{w}^{2}-\sum_{i=1}^{n-1} \mathrm{k}_{i}^{2}}, \mathrm{k}_{i} \neq 0$ for $i=1, \ldots, n$. The way of choosing separation constants is not unique. In this paper $k_{i}, i=1, \ldots, n-1$ are selected according to a branching rule method [19, 24, 28], see next sections.

\section{Three types of boundary conditions.}

D: A Dirichlet boundary condition defines the value of the function itself

$$
\Psi(\mathbf{x})=f(\mathbf{x}), \text { for } \mathbf{x} \in \partial F
$$


where $f(\mathbf{x})$ is a given function defined on the boundary.

N: A Neumann boundary condition defines the value of the normal derivative of the function

$$
\frac{\partial \Psi}{\partial \mathbf{n}}(\mathbf{x})=f(\mathbf{x}), \text { for } \mathbf{x} \in \partial F,
$$

where $\mathbf{n}$ denotes normal vector to the boundary $\partial F$.

M: A mixed boundary condition defines the value of the function itself on one part of the boundary and the value of the normal derivative of the function on the other part of the boundary

$$
\text { D: }\left.\Psi\right|_{\partial F_{0}}=f_{0}, \quad \mathbf{N}:\left.\quad \frac{\partial \Psi}{\partial \mathbf{n}}\right|_{\partial F_{1}}=f_{1},
$$

where $\partial F=\partial F_{0} \cup \partial F_{1}$ and $f_{0}, f_{1}$ are given functions, defined on the appropriate boundary.

\section{Finite REFLECTION GROUPS}

Our method is general and can be presented for any crystallographic finite reflection groups $G$ of any rank and any dimension which are associated with simple and semisimple Lie algebras/groups [1, 7, 10, 27. There is a complete classification of finite reflection groups given by Dynkin diagrams [2, 3, 10. These graphs provide the relative angles and relative length of the vectors of a set of simple roots of the root systems. There are two kinds of root systems according to the number of roots with different lengths: systems with one root length, and systems with two root lengths. A reflection $r$ in a hyperplane orthogonal to the long/short root and passing through the origin of $\mathbb{R}^{n}$ be denoted by $r_{l} / r_{s}$ respectively.

Working with finite reflection groups, it is convenient to use four bases in $\mathbb{R}^{n}$, namely natural $e$-, the simple root $\alpha$-, co-root $\check{\alpha}$ - and weight $\omega$-bases [2, 7, 10, The co-root basis $\check{\alpha}$ is defined by the formula

$$
\check{\alpha}_{i}=\frac{2 \alpha_{i}}{\left\langle\alpha_{i} \mid \alpha_{i}\right\rangle} .
$$

The $\omega$-basis is dual to simple root basis. The relationship between considered bases is standard for group theory and is expressed by

$$
\left\langle\check{\alpha}_{i} \mid \omega_{j}\right\rangle=\delta_{i j}
$$

There are two types of fundamental region either simplex for simple Lie group $G$ or prism for semisimple one. The simplex with $n+1$ vertices has the following coordinates

$$
F=\left\{0, \frac{\omega_{1}}{q_{1}}, \ldots, \frac{\omega_{n}}{q_{n}}\right\}
$$

where $q_{i}, i=1, \ldots, n$, called co-marks, can be found in [6, 10] for any simple Lie group $G$ of any rank and any dimension. The fundamental region for prisms can be given in the following sense. Let $G=G_{1} \times$ $G_{2}$, where $G_{1}, G_{2}$ are finite reflection groups. Let $\omega_{1}, \ldots, \omega_{k}$ be a set of generating elements of $G_{1}$ and $\omega_{k+1}, \ldots, \omega_{n}$ of $G_{2}$. Then the prism can be written as follows

$$
F=\left\{0, \frac{\omega_{i}}{q_{i}}, \frac{\omega_{j}}{q_{j}}, \frac{\omega_{i}}{q_{i}}+\frac{\omega_{j}}{q_{j}}\right\}
$$

where $i=1, \ldots, k$ and $j=k+1, \ldots, n$ and $q_{i}, q_{j}$ are co-marks [6, 10].

Let $\partial F_{i}$ be contained in the hyperplane generated by a set of orthogonal reflections

$$
r_{0}, r_{1}, \ldots, r_{i-1}, r_{i+1}, \ldots, r_{n}
$$

$i=\{0, \ldots, n\}$, where $r_{0}$ is an affine reflection (it corresponds to long reflection). If $r_{i}$ corresponds to the reflection orthogonal to the short/long root then we denote a part of the boundary by $\partial F_{s}$ or $\partial F_{l}$ respectively. In other words we can say that the boundary $\partial F$ of the fundamental region $F$ will be denoted by $\partial F_{l} / \partial F_{s}$ if its normal vector is perpendicular to the long/short root $\alpha$ respectively.

\section{Special Functions as a solution of Helmholtz EQUATION}

There are four kinds of special functions of interest to us whose orthogonality on lattice fragment $F$ is known for any simple Lie group [5, 6, 10, 16, 17, 19. 20, 23, and references therein]. The general formula for special functions (called orbit functions) [10, 11] corresponding to the finite reflection group $G$ is given by

$$
\sum_{w \in G} \sigma(w) e^{2 \pi i\langle w \lambda \mid \mathbf{x}\rangle}, \quad \lambda \in P^{+}, x \in F
$$

where the summation extends over the whole group $G, P^{+}$denotes the set of dominant weights [10] and $\sigma(w)= \pm 1$ depends on the type of the orbit function. The homomorphism $\sigma: G \rightarrow\{ \pm 1\}$ is a product of $\sigma\left(r_{l}\right), \sigma\left(r_{s}\right) \in\{ \pm 1\}$. There are four types of maps $\sigma$ [16, 17]:

$$
\begin{aligned}
\sigma\left(r_{l}\right)=\sigma\left(r_{s}\right)=1 & \Longrightarrow C, \\
\sigma\left(r_{l}\right)=\sigma\left(r_{s}\right)=-1 & \Longrightarrow S, \\
\sigma\left(r_{l}\right)=-1, \quad \sigma\left(r_{s}\right)=1 & \Longrightarrow S^{l}, \\
\sigma\left(r_{l}\right)=1, \quad \sigma\left(r_{s}\right)=-1 & \Longrightarrow S^{s} .
\end{aligned}
$$

All four families of functions defined above are formed as finite sums of exponential terms. The first two families, namely $C$ - and $S$-functions are generalized cosine and sine functions. They are symmetric and skew-symmetric with respect to the finite reflection group [6, 10, 16, 19, 21, 23. The other two, $S^{s}$ - and $S^{l}$-functions [11, 12, 16, 17, 23] have analogous properties as $C$ - and $S$-functions. The main difference between them is their behaviour at the boundary of their domain of orthogonality in $\mathbb{R}^{n}$.

Every finite group $G$ generated by reflections can be reduced to a subgroup $A_{1} \times \cdots \times A_{1}$ using a branching 
rule method described in 13, 14, 19, 22, 24, 28. This method allows us to do the separation of variables for special functions (5) corresponding to group $G$. As a result, we have all the functions written as a product of sine and cosine functions.

Remark 1. All four families of functions (5) presented above are solutions of the Helmholtz equation (1) where $\mathrm{w}^{2}=4 \pi^{2}\langle\lambda \mid \lambda\rangle$ with one of the three types of boundary conditions described in $\$ 2$.

Projection matrix reduces any $n$-dimensional group $G$ to a subgroup $A_{1} \times \ldots \times A_{1}$ [13, 19]. The branching rule allows one to divide any orbit of group $G$ into a union of orbits of group $A_{1}$. As an example see 3D cases described in $\S 5$.

Remark 2. The union of orbits which we get after reduction determine our choice of separating constants used in solution of Helmholtz equation (1).

The behaviour of the functions $C, S, S^{s}$ and $S^{l}$ on the boundary $\partial F$ can be summarize in the Tab. 1 .

\begin{tabular}{cccccc}
\hline & \multicolumn{2}{c}{$\mathbf{D}$} & & \multicolumn{2}{c}{$\mathbf{N}$} \\
\cline { 2 - 3 } \cline { 5 - 6 } & $\partial F_{s}$ & $\partial F_{l}$ & & $\partial F_{s}$ & $\partial F_{l}$ \\
\hline$C_{\lambda}(\mathbf{x})$ & $*$ & $*$ & & 0 & 0 \\
$S_{\lambda}(\mathbf{x})$ & 0 & 0 & $*$ & $*$ \\
\hline$S_{\lambda}^{s}(\mathbf{x})$ & 0 & $*$ & $*$ & 0 \\
$S_{\lambda}^{l}(\mathbf{x})$ & $*$ & 0 & & 0 & $*$ \\
\hline
\end{tabular}

TABle 1. Behaviour of the functions $C, S, S^{s}$ and $S^{l}$ on the boundary $\partial F$ for any finite refleciton group $G$ where $*$ denotes any function non-equivalent to 0 .

For any group $G$ considered in the paper $C$ functions fulfil the Dirichlet condition with value nonequivalent to 0 and the Neumann condition with 0 value on the whole boundary. The $S$-functions behave inversely. The $S^{s}$-functions fulfil the Dirichlet condition with a value non-equivalent to 0 on the part of boundary denoted by $\partial F_{l}$ and the Neumann condition with a value non-equivalent to 0 on the part of the boundary denoted by $\partial F_{s}$. The $S^{l}$ functions behave inversely. In the case of $C$-functions we talk about Dirichlet boundary condition and $S$-functions - Neumann boundary condition. For $S^{s}$ - and $S^{l}$-functions we talk about mixed boundary condition. In the next section we present $3 \mathrm{D}$ cases in details.

\section{3D FINITE REFLECTION GROUPS}

The 3 dimensional groups which we considered here are $B_{3}, C_{3}, C_{2} \times A_{1}, G_{2} \times A_{1}, A_{1} \times A_{1} \times A_{1}$ [2, 7, 8, 10]. We use the following notation for coordinates:

$$
\begin{aligned}
& \mathbb{R}^{3} \ni \lambda=(a, b, c)_{\omega}=a \omega_{1}+b \omega_{2}+c \omega_{3}, \\
& \mathbb{R}^{3} \ni \mathbf{x}=\left(x_{1}, x_{2}, x_{3}\right)_{\check{\alpha}}=\left(y_{1}, y_{2}, y_{3}\right)_{e},
\end{aligned}
$$

where indexes $e, \omega$, and $\check{\alpha}$ denote natural-, $\omega$-, and $\check{\alpha}$ basis, respectively. The action of the Laplace operator $\nabla$ on the functions given in different bases can be found in [10]. In the next subsections we describe each case in details.

For each case we present functions which are the solutions of Helmholtz equation (1). We give the exact forms of the projection matrices and branching rules which allow us to choose the separation constants used in (3). All functions described below fulfil one of the three types of boundary conditions described in $\$ 2$

\section{1. $B_{3}$ AND $C_{3}$ GROUPS}

The $\alpha$-basis vectors in Cartesian coordinates are

$$
\begin{aligned}
B_{3}: & C_{3}: \\
\alpha_{1}:=(1,-1,0)_{e}, & \alpha_{1}:=\frac{1}{\sqrt{2}}(1,-1,0)_{e}, \\
\alpha_{2}:=(0,1,-1)_{e}, & \alpha_{2}:=\frac{1}{\sqrt{2}}(0,1,-1)_{e}, \\
\alpha_{3}:=(0,0,1)_{e}, & \alpha_{3}:=\frac{1}{\sqrt{2}}(0,0,2)_{e} .
\end{aligned}
$$

As one can easily notice the short root for $B_{3}$ is $\alpha_{3}$ and for $C_{3}$ are $\alpha_{1}, \alpha_{2}$. The fundamental regions $F$ for $B_{3}$ and $C_{3}$ groups, written in $\omega$-basis, have the vertices:

$$
\begin{aligned}
& F_{B_{3}}=\left\{0, \omega_{1}, \frac{1}{2} \omega_{2}, \omega_{3}\right\}, \\
& F_{C_{3}}=\left\{0, \omega_{1}, \omega_{2}, \omega_{3}\right\},
\end{aligned}
$$

and are shown in Fig. 1 .

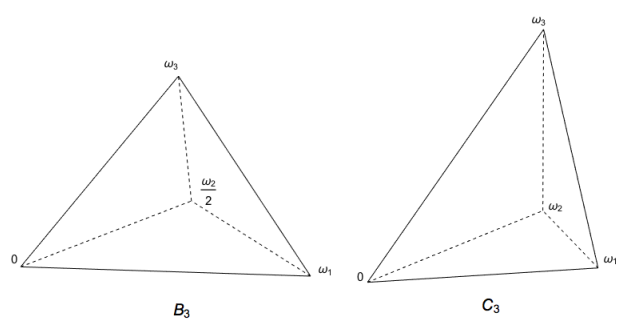

Figure 1. The fundamental region $F$ for $B_{3}$ and $C_{3}$ group.

The reduction of $B_{3}$ and $C_{3}$ to a subgroup $A_{1} \times$ $A_{1} \times A_{1}$ is given by the projection matrices

$$
P_{B_{3}}=\left(\begin{array}{ccc}
1 & 1 & 0 \\
1 & 1 & 1 \\
0 & 2 & 1
\end{array}\right), \quad P_{C_{3}}=\left(\begin{array}{ccc}
1 & 1 & 1 \\
0 & 1 & 1 \\
0 & 0 & 1
\end{array}\right) .
$$

Then the branching rule is the following:

$$
\begin{gathered}
O(a, b, c) \stackrel{P_{B_{3}}}{\longrightarrow} O(2 a+2 b+c) O(2 b+c) O(c) \\
\cup O(2 b+c) O(2 a+2 b+c) O(c) \\
\cup O(2 a+2 b+c) O(c) O(2 b+c) \\
\cup O(c) O(2 a+2 b+c) O(2 b+c) \\
\cup O(2 b+c) O(c) O(2 a+2 b+c) \\
\cup O(c) O(2 b+c) O(2 a+2 b+c),
\end{gathered}
$$




$$
\begin{aligned}
O(a, b, c) \stackrel{P_{C_{3}}}{\longrightarrow} O(a+b+c) O(b+c) O(c) \\
\cup O(b+c) O(a+b+c) O(c) \cup O(a+b+c) O(c) O(b+c) \\
\cup O(b+c) O(c) O(a+b+c) \cup O(c) O(a+b+c) O(b+c) \\
\cup O(c) O(b+c) O(a+b+c) .
\end{aligned}
$$

According to Remarks 1 and 2 the separation constants for $B_{3}$ and $C_{3}$ group we can choose as

$$
\begin{aligned}
-k_{1}^{2} & =-\pi^{2}(2 a+2 b+c)^{2}, \\
-k_{2}^{2} & =-\pi^{2}(2 b+c)^{2}, \\
-k_{3}^{2} & =-\pi^{2} c^{2}
\end{aligned}
$$

where $\mathrm{w}^{2}=4 \pi^{2}\left(a^{2}+2 a b+2 b^{2}+a c+2 b c+\frac{3}{4} c^{2}\right)$. The separation constants for $C_{3}$ group are

$$
\begin{aligned}
-k_{1}^{2} & =-\pi^{2}(a+b+c)^{2}, \\
-k_{2}^{2} & =-\pi^{2}(b+c)^{2}, \\
-k_{3}^{2} & =-\pi^{2} c^{2},
\end{aligned}
$$

where $\mathrm{w}^{2}=4 \pi^{2}\left(\frac{1}{2} a^{2}+a b+b^{2}+a c+2 b c+\frac{3}{2} c^{2}\right)$.

The explicit forms of orbit functions for $B_{3}$ and $C_{3}$ group have form

$$
\begin{aligned}
& B_{3}: \quad C_{a, b, c}(\mathbf{x})=C_{2 a+2 b+c}\left(\mathrm{x}_{1}\right) C_{2 b+c}\left(\mathrm{x}_{2}\right) C_{c}\left(\mathrm{x}_{3}\right) \\
& +C_{2 b+c}\left(\mathrm{x}_{1}\right) C_{2 a+2 b+c}\left(\mathrm{x}_{2}\right) C_{c}\left(\mathrm{x}_{3}\right) \\
& +C_{2 a+2 b+c}\left(\mathrm{x}_{1}\right) C_{c}\left(\mathrm{x}_{2}\right) C_{2 b+c}\left(\mathrm{x}_{3}\right) \\
& +C_{c}\left(\mathrm{x}_{1}\right) C_{2 a+2 b+c}\left(\mathrm{x}_{2}\right) C_{2 b+c}\left(\mathrm{x}_{3}\right) \\
& +C_{2 b+c}\left(\mathrm{x}_{1}\right) C_{c}\left(\mathrm{x}_{2}\right) C_{2 a+2 b+c}\left(\mathrm{x}_{3}\right) \\
& +C_{c}\left(\mathrm{x}_{1}\right) C_{2 b+c}\left(\mathrm{x}_{2}\right) C_{2 a+2 b+c}\left(\mathrm{x}_{3}\right), \\
& S_{a, b, c}^{l}(\mathbf{x})=C_{2 a+2 b+c}\left(\mathrm{x}_{1}\right) C_{2 b+c}\left(\mathrm{x}_{2}\right) C_{c}\left(\mathrm{x}_{3}\right) \\
& -C_{2 b+c}\left(\mathrm{x}_{1}\right) C_{2 a+2 b+c}\left(\mathrm{x}_{2}\right) C_{c}\left(\mathrm{x}_{3}\right) \\
& -C_{2 a+2 b+c}\left(\mathrm{x}_{1}\right) C_{c}\left(\mathrm{x}_{2}\right) C_{2 b+c}\left(\mathrm{x}_{3}\right) \\
& +C_{c}\left(\mathrm{x}_{1}\right) C_{2 a+2 b+c}\left(\mathrm{x}_{2}\right) C_{2 b+c}\left(\mathrm{x}_{3}\right) \\
& +C_{2 b+c}\left(\mathrm{x}_{1}\right) C_{c}\left(\mathrm{x}_{2}\right) C_{2 a+2 b+c}\left(\mathrm{x}_{3}\right) \\
& -C_{c}\left(\mathrm{x}_{1}\right) C_{2 b+c}\left(\mathrm{x}_{2}\right) C_{2 a+2 b+c}\left(\mathrm{x}_{3}\right), \\
& \begin{aligned}
S_{a, b, c}(\mathrm{x}) & =S_{2 a+2 b+c}\left(\mathrm{x}_{1}\right) S_{2 b+c}\left(\mathrm{x}_{2}\right) S_{c}\left(\mathrm{x}_{3}\right) \\
& -S_{2 b+c}\left(\mathrm{x}_{1}\right) S_{2 a+2 b+c}\left(\mathrm{x}_{2}\right) S_{c}\left(\mathrm{x}_{3}\right) \\
& -S_{2 a+2 b+c}\left(\mathrm{x}_{1}\right) S_{c}\left(\mathrm{x}_{2}\right) S_{2 b+c}\left(\mathrm{x}_{3}\right) \\
& +S_{c}\left(\mathrm{x}_{1}\right) S_{2 a+2 b+c}\left(\mathrm{x}_{2}\right) S_{2 b+c}\left(\mathrm{x}_{3}\right) \\
& +S_{2 b+c}\left(\mathrm{x}_{1}\right) S_{c}\left(\mathrm{x}_{2}\right) S_{2 a+2 b+c}\left(\mathrm{x}_{3}\right) \\
& -S_{c}\left(\mathrm{x}_{1}\right) S_{2 b+c}\left(\mathrm{x}_{2}\right) S_{2 a+2 b+c}\left(\mathrm{x}_{3}\right)
\end{aligned} \\
& S_{a, b, c}^{s}(\mathbf{x})=S_{2 a+2 b+c}\left(\mathrm{x}_{1}\right) S_{2 b+c}\left(\mathrm{x}_{2}\right) S_{c}\left(\mathrm{x}_{3}\right) \\
& +S_{2 b+c}\left(\mathrm{x}_{1}\right) S_{2 a+2 b+c}\left(\mathrm{x}_{2}\right) S_{c}\left(\mathrm{x}_{3}\right) \\
& +S_{2 a+2 b+c}\left(\mathrm{x}_{1}\right) S_{c}\left(\mathrm{x}_{2}\right) S_{2 b+c}\left(\mathrm{x}_{3}\right) \\
& +S_{c}\left(\mathrm{x}_{1}\right) S_{2 a+2 b+c}\left(\mathrm{x}_{2}\right) S_{2 b+c}\left(\mathrm{x}_{3}\right) \\
& +S_{2 b+c}\left(\mathrm{x}_{1}\right) S_{c}\left(\mathrm{x}_{2}\right) S_{2 a+2 b+c}\left(\mathrm{x}_{3}\right) \\
& +S_{c}\left(\mathrm{x}_{1}\right) S_{2 b+c}\left(\mathrm{x}_{2}\right) S_{2 a+2 b+c}\left(\mathrm{x}_{3}\right) \text {; }
\end{aligned}
$$

$$
\begin{aligned}
C_{3}: C_{a, b, c}(\mathrm{x}) & =C_{a+b+c}\left(\mathrm{x}_{1}\right) C_{b+c}\left(\mathrm{x}_{2}\right) C_{c}\left(\mathrm{x}_{3}\right) \\
& +C_{b+c}\left(\mathrm{x}_{1}\right) C_{a+b+c}\left(\mathrm{x}_{2}\right) C_{c}\left(\mathrm{x}_{3}\right) \\
& +C_{a+b+c}\left(\mathrm{x}_{1}\right) C_{c}\left(\mathrm{x}_{2}\right) C_{b+c}\left(\mathrm{x}_{3}\right) \\
& +C_{c}\left(\mathrm{x}_{1}\right) C_{a+b+c}\left(\mathrm{x}_{2}\right) C_{b+c}\left(\mathrm{x}_{3}\right) \\
& +C_{b+c}\left(\mathrm{x}_{1}\right) C_{c}\left(\mathrm{x}_{2}\right) C_{a+b+c}\left(\mathrm{x}_{3}\right) \\
& +C_{c}\left(\mathrm{x}_{1}\right) C_{b+c}\left(\mathrm{x}_{2}\right) C_{a+b+c}\left(\mathrm{x}_{3}\right),
\end{aligned}
$$

$$
\begin{aligned}
S_{a, b, c}^{s}(\mathrm{x}) & =C_{a+b+c}\left(\mathrm{x}_{1}\right) C_{b+c}\left(\mathrm{x}_{2}\right) C_{c}\left(\mathrm{x}_{3}\right) \\
& -C_{b+c}\left(\mathrm{x}_{1}\right) C_{a+b+c}\left(\mathrm{x}_{2}\right) C_{c}\left(\mathrm{x}_{3}\right) \\
& -C_{a+b+c}\left(\mathrm{x}_{1}\right) C_{c}\left(\mathrm{x}_{2}\right) C_{b+c}\left(\mathrm{x}_{3}\right) \\
& +C_{c}\left(\mathrm{x}_{1}\right) C_{a+b+c}\left(\mathrm{x}_{2}\right) C_{b+c}\left(\mathrm{x}_{3}\right) \\
& +C_{b+c}\left(\mathrm{x}_{1}\right) C_{c}\left(\mathrm{x}_{2}\right) C_{a+b+c}\left(\mathrm{x}_{3}\right) \\
& -C_{c}\left(\mathrm{x}_{1}\right) C_{b+c}\left(\mathrm{x}_{2}\right) C_{a+b+c}\left(\mathrm{x}_{3}\right),
\end{aligned}
$$

$$
\begin{aligned}
S_{a, b, c}(\mathrm{x}) & =S_{a+b+c}\left(\mathrm{x}_{1}\right) S_{b+c}\left(\mathrm{x}_{2}\right) S_{c}\left(\mathrm{x}_{3}\right) \\
& -S_{b+c}\left(\mathrm{x}_{1}\right) S_{a+b+c}\left(\mathrm{x}_{2}\right) S_{c}\left(\mathrm{x}_{3}\right) \\
& -S_{a+b+c}\left(\mathrm{x}_{1}\right) S_{c}\left(\mathrm{x}_{2}\right) S_{b+c}\left(\mathrm{x}_{3}\right) \\
& +S_{c}\left(\mathrm{x}_{1}\right) S_{a+b+c}\left(\mathrm{x}_{2}\right) S_{b+c}\left(\mathrm{x}_{3}\right) \\
& +S_{b+c}\left(\mathrm{x}_{1}\right) S_{c}\left(\mathrm{x}_{2}\right) S_{a+b+c}\left(\mathrm{x}_{3}\right) \\
& -S_{c}\left(\mathrm{x}_{1}\right) S_{b+c}\left(\mathrm{x}_{2}\right) S_{a+b+c}\left(\mathrm{x}_{3}\right)
\end{aligned}
$$

$$
\begin{aligned}
S_{a, b, c}^{l}(\mathrm{x}) & =S_{a+b+c}\left(\mathrm{x}_{1}\right) S_{b+c}\left(\mathrm{x}_{2}\right) S_{c}\left(\mathrm{x}_{3}\right) \\
& +S_{b+c}\left(\mathrm{x}_{1}\right) S_{a+b+c}\left(\mathrm{x}_{2}\right) S_{c}\left(\mathrm{x}_{3}\right) \\
& +S_{a+b+c}\left(\mathrm{x}_{1}\right) S_{c}\left(\mathrm{x}_{2}\right) S_{b+c}\left(\mathrm{x}_{3}\right) \\
& +S_{c}\left(\mathrm{x}_{1}\right) S_{a+b+c}\left(\mathrm{x}_{2}\right) S_{b+c}\left(\mathrm{x}_{3}\right) \\
& +S_{b+c}\left(\mathrm{x}_{1}\right) S_{c}\left(\mathrm{x}_{2}\right) S_{a+b+c}\left(\mathrm{x}_{3}\right) \\
& +S_{c}\left(\mathrm{x}_{1}\right) S_{b+c}\left(\mathrm{x}_{2}\right) S_{a+b+c}\left(\mathrm{x}_{3}\right) .
\end{aligned}
$$

The functions on the right side of the above equations are special functions corresponding to group $A_{1}$

$$
\begin{aligned}
& C_{\mu}\left(\mathrm{x}_{i}\right)=\sum_{\mu \in A_{1}} e^{2 \pi i\left\langle\mu \mid \mathrm{x}_{i}\right\rangle}=2 \cos \left(2 \pi \mu \mathrm{x}_{i}\right), \\
& S_{\mu}\left(\mathrm{x}_{i}\right)=\sum_{\mu \in A_{1}} \sigma(\mu) e^{2 \pi i\left\langle\mu \mid \mathrm{x}_{i}\right\rangle}=2 i \sin \left(2 \pi \mu \mathrm{x}_{i}\right),
\end{aligned}
$$

where $\mu \in P_{A_{1}}^{+}, \mathrm{x}_{i} \in F_{A_{1}}, i=1,2,3$. The coordinate $\mathrm{x}_{i}$ respond to the $i$-th coordinate in $A_{1} \times A_{1} \times A_{1}$. The functions $C_{\mu}\left(\mathrm{x}_{i}\right)$ and $S_{\mu}\left(\mathrm{x}_{i}\right)$ are the solutions of Helmholtz equation (1) in the form (3) in 1D case.

For group $B_{3}$ the functions $C$ - and $S^{l}$ - are real valued and $S$ - and $S^{s}$ are purely imaginary. In the case of $C_{3}$, the functions $C$ - and $S^{s}$ - are real valued and $S$ - and $S^{l}$ are purely imaginary.

The normal vectors shown on Fig. 2 are

$$
\begin{array}{ccrl}
B_{3}: & C_{3}: \\
n_{1}=\left\{-\frac{1}{\sqrt{2}}, \frac{1}{\sqrt{2}}, 0\right\}, & & n_{1}=\{0,0,1\}, \\
n_{2}=\left\{0,-\frac{1}{\sqrt{2}}, \frac{1}{\sqrt{2}}\right\}, & & n_{2}=\left\{0, \frac{1}{\sqrt{2}},-\frac{1}{\sqrt{2}}\right\}, \\
n_{3}=\{0,0,-1\}, & & n_{3}=\left\{\frac{1}{\sqrt{2}},-\frac{1}{\sqrt{2}}, 0\right\}, \\
n_{4}=\left\{\frac{1}{\sqrt{2}}, \frac{1}{\sqrt{2}}, 0\right\}, & & n_{4}=\{1,0,0\} .
\end{array}
$$




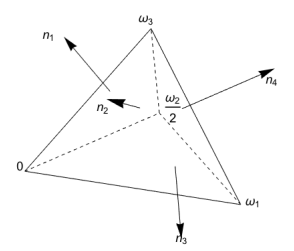

$B_{3}$

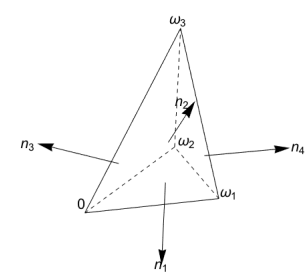

$C_{3}$
Figure 2. Normal vectors for $B_{3}$ and $C_{3}$.

In the case of $B_{3}$ group normal vector $n_{4}$ is perpendicular to the short simple root. The rest of them, namely $n_{1}, n_{2}, n_{3}$ are perpendicular to the long simple roots. So the boundaries that correspond to normal vectors are $\partial F_{s}$ for $n_{1}$ and $\partial F_{l}$ for the others. The values of the functions on the boundaries are summarized in the Appendix in Tab. 2. In the case of $C_{3}$ it is a little bit different, the normal vectors $n_{2}, n_{3}$ are perpendicular to the short simple roots and $n_{1}, n_{4}$ - to the long simple roots. The values of the functions on the boundaries are given in the Appendix in Tab. 3

\section{2. $C_{2} \times A_{1}$ AND $G_{2} \times A_{1}$ GROUPS}

The $\alpha$-basis vectors in Cartesian coordinates have the form

$$
\begin{array}{rlrl}
C_{2} & \times A_{1}: & G_{2} \times A_{1}: \\
\alpha_{1}:=\frac{1}{\sqrt{2}}(1,-1,0)_{e}, & \alpha_{1}:=(\sqrt{2}, 0,0)_{e}, \\
\alpha_{2}:=\frac{2}{\sqrt{2}}(0,2,0)_{e}, & \alpha_{2}:=\left(-\frac{1}{\sqrt{2}}, \frac{1}{\sqrt{6}}, 0\right)_{e}, \\
\alpha_{3}:=\frac{1}{\sqrt{2}}(0,0,2)_{e}, & \alpha_{3}:=\frac{1}{\sqrt{2}}(0,0,2)_{e} .
\end{array}
$$

The vertices of the fundamental regions $F$ for $C_{2} \times$ $A_{1}, G_{2} \times A_{1}$ groups, shown in Fig. 3 , written in $\omega$-basis are

$$
\begin{aligned}
& F_{C_{2} \times A_{1}}=\left\{0, \omega_{1}, \omega_{2}, \omega_{3}, \omega_{1}+\omega_{3}, \omega_{2}+\omega_{3}\right\} \\
& F_{G_{2} \times A_{1}}=\left\{0, \frac{1}{2} \omega_{1}, \omega_{2}, \omega_{3}, \frac{1}{2} \omega_{1}+\omega_{3}, \omega_{2}+\omega_{3}\right\}
\end{aligned}
$$
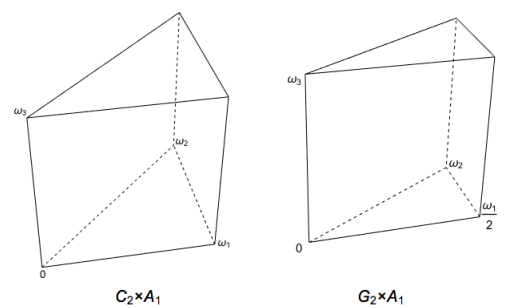

Figure 3. The fundamental region $F$ for $C_{2} \times A_{1}$ and $G_{2} \times A_{1}$ group.
The groups $C_{2} \times A_{1}, G_{2} \times A_{1}$ can be reduced to a subgroup $A_{1} \times A_{1} \times A_{1}$ using a branching rule method described in [19, 24].

The projection matrices and the branching rules are

$$
\begin{gathered}
P_{C_{2} \times A_{1}}=\left(\begin{array}{ccc}
1 & 1 & 0 \\
0 & 1 & 0 \\
0 & 0 & 1
\end{array}\right), \quad P_{G_{2} \times A_{1}}=\left(\begin{array}{lll}
1 & 1 & 0 \\
3 & 1 & 0 \\
0 & 0 & 1
\end{array}\right), \\
O(a, b) O(c) \stackrel{P_{C_{2} \times A_{1}}}{\longrightarrow} \\
\cup(a+b) O(b) O(c) \\
\cup O(b) O(a+b) O(c), \\
O(a, b) O(c) \stackrel{P_{G_{2} \times A_{1}}}{\longrightarrow} O(a+b) O(3 a+b) O(c) \\
\cup O(2 a+b) O(b) O(c) \cup O(a) O(3 a+2 b) O(c) .
\end{gathered}
$$

The separation constants for $C_{2} \times A_{1}$ are

$-k_{1}^{2}=-\pi^{2}(a+b)^{2}, \quad-k_{2}^{2}=-\pi^{2} b^{2}, \quad-k_{3}^{2}=-\pi^{2} c^{2}$,

where $\mathrm{w}^{2}=4 \pi^{2}\left(a^{2}+a b+b^{2}+\frac{1}{2} c^{2}\right)$ and for $G_{2} \times A_{1}$ equal

$$
\begin{array}{rlrl}
-k_{1}^{2} & =-2 \pi^{2}(2 a+b)^{2}, & & -l_{1}^{2}=-2 \pi^{2}(a+b)^{2}, \\
-k_{2}^{2} & =-\frac{2}{3} \pi^{2} b^{2}, & & -l_{2}^{2}=-\frac{2}{3} \pi^{2}(3 a+b)^{2}, \\
-k_{3}^{2} & =-2 \pi^{2} c^{2}, & -l_{3}^{2}=-2 \pi^{2} c^{2}, \\
-m_{1}^{2} & =-2 \pi^{2} a^{2}, & \\
-m_{2}^{2} & =-\frac{2}{3} \pi^{2}(3 a+2 b)^{2}, & \\
-m_{3}^{2} & =-2 \pi^{2} c^{2}, &
\end{array}
$$

where $\mathrm{w}^{2}=4 \pi^{2}\left(2 a^{2}+2 a b+\frac{2}{3} b^{2}+\frac{1}{2} c^{2}\right)$.

The explicit forms of orbit functions are $C_{2} \times A_{1}$ :

$$
\begin{aligned}
C_{a, b, c}(\mathrm{x})= & C_{a+b}\left(\mathrm{x}_{1}\right) C_{b}\left(\mathrm{x}_{2}\right) C_{c}\left(\mathrm{x}_{3}\right) \\
& +C_{b}\left(\mathrm{x}_{1}\right) C_{a+b}\left(\mathrm{x}_{2}\right) C_{c}\left(\mathrm{x}_{3}\right), \\
S_{a, b, c}^{s}(\mathbf{x})= & C_{a+b}\left(\mathrm{x}_{1}\right) C_{b}\left(\mathrm{x}_{2}\right) C_{c}\left(\mathrm{x}_{3}\right) \\
& -C_{b}\left(\mathrm{x}_{1}\right) C_{a+b}\left(\mathrm{x}_{2}\right) C_{c}\left(\mathrm{x}_{3}\right), \\
S_{a, b, c}(\mathrm{x})= & S_{a+b}\left(\mathrm{x}_{1}\right) S_{b}\left(\mathrm{x}_{2}\right) S_{c}\left(\mathrm{x}_{3}\right) \\
& -S_{b}\left(\mathrm{x}_{1}\right) S_{a+b}\left(\mathrm{x}_{2}\right) S_{c}\left(\mathrm{x}_{3}\right), \\
S_{a, b, c}^{l}(\mathbf{x})= & S_{a+b}\left(\mathrm{x}_{1}\right) S_{b}\left(\mathrm{x}_{2}\right) S_{c}\left(\mathrm{x}_{3}\right) \\
& +S_{b}\left(\mathrm{x}_{1}\right) S_{a+b}\left(\mathrm{x}_{2}\right) S_{c}\left(\mathrm{x}_{3}\right)
\end{aligned}
$$

$G_{2} \times A_{1}$

$$
\begin{gathered}
C_{a, b, c}(\mathrm{x})=C_{a}\left(\mathrm{x}_{1}\right) C_{3 a+2 b}\left(\mathrm{x}_{2}\right) C_{c}\left(\mathrm{x}_{3}\right) \\
+C_{a+b}\left(\mathrm{x}_{1}\right) C_{3 a+b}\left(\mathrm{x}_{2}\right) C_{c}\left(\mathrm{x}_{3}\right) \\
+C_{2 a+b}\left(\mathrm{x}_{1}\right) C_{b}\left(\mathrm{x}_{2}\right) C_{c}\left(\mathrm{x}_{3}\right), \\
S_{a, b, c}^{l}(\mathrm{x})=S_{a}\left(\mathrm{x}_{1}\right) C_{3 a+2 b}\left(\mathrm{x}_{2}\right) S_{c}\left(\mathrm{x}_{3}\right) \\
-S_{a+b}\left(\mathrm{x}_{1}\right) C_{3 a+b}\left(\mathrm{x}_{2}\right) S_{c}\left(\mathrm{x}_{3}\right) \\
+S_{2 a+b}\left(\mathrm{x}_{1}\right) C_{b}\left(\mathrm{x}_{2}\right) S_{c}\left(\mathrm{x}_{3}\right), \\
S_{a, b, c}(\mathbf{x})=S_{a}\left(\mathrm{x}_{1}\right) S_{3 a+2 b}\left(\mathrm{x}_{2}\right) S_{c}\left(\mathrm{x}_{3}\right) \\
-S_{a+b}\left(\mathrm{x}_{1}\right) S_{3 a+b}\left(\mathrm{x}_{2}\right) S_{c}\left(\mathrm{x}_{3}\right) \\
+S_{2 a+b}\left(\mathrm{x}_{1}\right) S_{b}\left(\mathrm{x}_{2}\right) S_{c}\left(\mathrm{x}_{3}\right)
\end{gathered}
$$




$$
\begin{gathered}
S_{a, b, c}^{s}(\mathrm{x})=C_{a}\left(\mathrm{x}_{1}\right) S_{3 a+2 b}\left(\mathrm{x}_{2}\right) C_{c}\left(\mathrm{x}_{3}\right) \\
-C_{a+b}\left(\mathrm{x}_{1}\right) S_{3 a+b}\left(\mathrm{x}_{2}\right) C_{c}\left(\mathrm{x}_{3}\right) \\
-C_{2 a+b}\left(\mathrm{x}_{1}\right) S_{b}\left(\mathrm{x}_{2}\right) C_{c}\left(\mathrm{x}_{3}\right),
\end{gathered}
$$

where $C_{\mu}\left(\mathrm{x}_{i}\right), S_{\mu}\left(\mathrm{x}_{i}\right)$ for $i=1,2,3$ are the same as in the previous cases.

For group $C_{2} \times A_{1}$ the functions $C$ - and $S^{s}$ - are real valued and $S$ - and $S^{l}$ are purely imaginary. In the case of $G_{2} \times A_{1}$, the functions $C$ - and $S^{l}$ - are real valued and $S$ - and $S^{s}$ are purely imaginary.
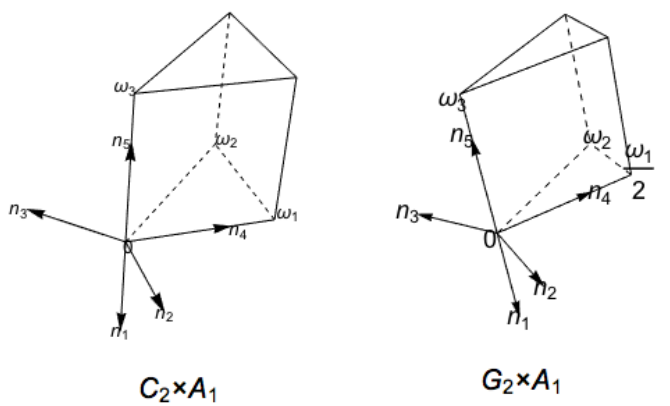

Figure 4. Normal vectors of $F$ for $C_{2} \times A_{1}, G_{2} \times A_{1}$ groups.

The normal vectors shown in Fig. 4 are

$$
\begin{array}{rlrl}
C_{2} \times A_{1}: & G_{2} \times A_{1}: \\
n_{1} & =\{0,0,-1\}, & & n_{1}=\{0,0,-1\}, \\
n_{2} & =\{0,-1,0\}, & & n_{2}=\left\{\frac{\sqrt{3}}{2},-\frac{1}{2}, 0\right\}, \\
n_{3} & =\left\{-\frac{1}{\sqrt{2}}, \frac{1}{\sqrt{2}}, 0\right\}, & & n_{3}=\{-1,0,0\}, \\
n_{4} & =\{1,0,0\}, & & n_{4}=\left\{\frac{1}{2}, \frac{\sqrt{3}}{2}, 0\right\}, \\
n_{5} & =\{0,0,1\}, & & n_{5}=\{0,0,1\} .
\end{array}
$$

In the case of $C_{2} \times A_{1}$ the group normal vector $n_{3}$ is perpendicular to the short simple root. The rest of them, namely $n_{1}, n_{2}, n_{4}, n_{5}$ are perpendicular to the long simple roots. So the boundaries that correspond to normal vectors are $\partial F_{s}$ for $n_{3}$ and $\partial F_{l}$ for the others. The values of the functions on the boundaries are summarized in Appendix in Tab. 4. In the case of $G_{2} \times A_{1}$, the normal vector $n_{2}$ corresponds to the short simple root so to the boundary $\partial F_{s}$ and the rest of normal vectors to the long simple roots i.e. to the boundaries $\partial F_{l}$. The values of the functions on the boundaries are given in Appendix in Tab. 5

\section{3. $A_{1} \times A_{1} \times A_{1}$ GROUP}

Although the root system of $A_{1} \times A_{1} \times A_{1}$ does not have two different lengths of roots, it is still an interesting case for us. The $\alpha$-basis vectors in Cartesian coordinates have the form

$$
\begin{gathered}
\alpha_{1}:=(\sqrt{2}, 0,0)_{e}, \quad \alpha_{2}:=(0, \sqrt{2}, 0)_{e}, \\
\alpha_{3}:=(0,0, \sqrt{2})_{e} .
\end{gathered}
$$

According to (4) and (5) there are two families of special functions $C$ and $S$. By the analogy to homomorphism (5) we can define new families of functions.

$$
\begin{aligned}
& \sigma\left(r_{1}\right)=\sigma\left(r_{2}\right)=\sigma\left(r_{3}\right)=1 \quad \Longrightarrow \quad C C C, \\
& \sigma\left(r_{1}\right)=\sigma\left(r_{2}\right)=\sigma\left(r_{3}\right)=-1 \quad \Longrightarrow \quad S S S, \\
& \sigma\left(r_{1}\right)=\sigma\left(r_{2}\right)=1, \quad \sigma\left(r_{3}\right)=-1 \quad \Longrightarrow \quad C C S \text {, } \\
& \sigma\left(r_{1}\right)=\sigma\left(r_{2}\right)=-1, \quad \sigma\left(r_{3}\right)=1 \quad \Longrightarrow \quad S S C \text {, } \\
& \sigma\left(r_{1}\right)=\sigma\left(r_{3}\right)=1, \quad \sigma\left(r_{2}\right)=-1 \quad \Longrightarrow \quad C S C, \\
& \sigma\left(r_{1}\right)=-1, \quad \sigma\left(r_{2}\right)=\sigma\left(r_{3}\right)=1 \quad \Longrightarrow \quad S C C, \\
& \sigma\left(r_{1}\right)=1, \quad \sigma\left(r_{2}\right)=\sigma\left(r_{3}\right)=-1 \quad \Longrightarrow \quad C S S \text {, } \\
& \sigma\left(r_{2}\right)=-1, \quad \sigma\left(r_{1}\right)=\sigma\left(r_{3}\right)=1 \quad \Longrightarrow \quad S C S,
\end{aligned}
$$

where $C C C, S S S$ correspond to $C$ and $S$-functions, respectively and the rest of them to $S^{l}$ - and $S^{s}$-functions. All families of functions defined on the fundamental region

$$
\begin{aligned}
& F_{A_{1} \times A_{1} \times A_{1}}=\left\{0, \omega_{1}, \omega_{2}, \omega_{3}, \omega_{1}+\omega_{2},\right. \\
&\left.\omega_{1}+\omega_{3}, \omega_{2}+\omega_{3}, \omega_{1}+\omega_{2}+\omega_{3}\right\} .
\end{aligned}
$$

fulfill mixed boundary condition (see Tab. 6).

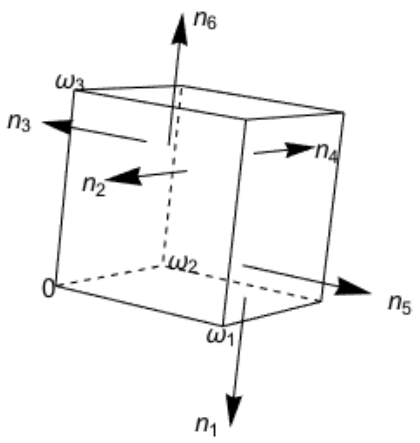

FIGURE 5. The fundamental region $F$ with normal vectors of $A_{1} \times A_{1} \times A_{1}$ group.

The projection matrix is the identity matrix and then the choice of separation constants is trivial:

$$
-k_{1}^{2}=-\pi^{2} a^{2}, \quad-k_{2}^{2}=-\pi^{2} b^{2}, \quad-k_{3}^{2}=-\pi^{2} c^{2} .
$$

According to the branching rule

$$
O(a, b, c) \stackrel{P_{A_{1} \times A_{1} \times A_{1}}}{\longrightarrow} O(a) O(b) O(c)
$$

we have

$$
\begin{aligned}
C C C_{a, b, c}(\mathbf{x}) & :=C_{a}\left(\mathrm{x}_{1}\right) C_{b}\left(\mathrm{x}_{2}\right) C_{c}\left(\mathrm{x}_{3}\right) \\
S C S_{a, b, c}(\mathbf{x}) & :=S_{a}\left(\mathrm{x}_{1}\right) C_{b}\left(\mathrm{x}_{2}\right) S_{c}\left(\mathrm{x}_{3}\right) \\
C S S_{a, b, c}(\mathbf{x}) & :=C_{a}\left(\mathrm{x}_{1}\right) S_{b}\left(\mathrm{x}_{2}\right) S_{c}\left(\mathrm{x}_{3}\right) \\
S S C_{a, b, c}(\mathbf{x}) & :=S_{a}\left(\mathrm{x}_{1}\right) S_{b}\left(\mathrm{x}_{2}\right) C_{c}\left(\mathrm{x}_{3}\right) \\
S S S_{a, b, c}(\mathbf{x}) & :=S_{a}\left(\mathrm{x}_{1}\right) S_{b}\left(\mathrm{x}_{2}\right) S_{c}\left(\mathrm{x}_{3}\right) \\
C S C_{a, b, c}(\mathbf{x}) & :=C_{a}\left(\mathrm{x}_{1}\right) S_{b}\left(\mathrm{x}_{2}\right) C_{c}\left(\mathrm{x}_{3}\right) \\
C C S_{a, b, c}(\mathbf{x}) & :=C_{a}\left(\mathrm{x}_{1}\right) C_{b}\left(\mathrm{x}_{2}\right) S_{c}\left(\mathrm{x}_{3}\right) \\
S C C_{a, b, c}(\mathbf{x}) & :=S_{a}\left(\mathrm{x}_{1}\right) C_{b}\left(\mathrm{x}_{2}\right) C_{c}\left(\mathrm{x}_{3}\right)
\end{aligned}
$$


where $C_{\mu}\left(\mathrm{x}_{i}\right), S_{\mu}\left(\mathrm{x}_{i}\right)$ for $i=1,2,3$ are the same as in the previous cases. The first four families of functions are real valued and the rest of them are pure imaginary.

Normal vectors shown on Fig. 5 are

$$
\begin{array}{ll}
n_{1}=\{0,0,-1\}, & n_{2}=\{0,-1,0\}, \\
n_{3}=\{-1,0,0\}, & n_{4}=\{0,1,0\}, \\
n_{5}=\{1,0,0\}, & n_{6}=\{0,0,1\} .
\end{array}
$$

The values of the functions on the boundaries are shown in Appendix in Tab. 6 .

\section{Appendix}

In Tables 26 we collect the values of special functions on the boundaries of the fundamental region $F$ for each of $3 \mathrm{D}$ finite reflection groups presented in the paper.

\section{REFERENCES}

[1] Borel, A., and J. de Siebental, "Les sous-groupes fermés de rang maximum de groupes de Lie clos." Comment. Math. Helv. 23, (1949): 200-221.

[2] Bourbaki, N. Groupes et algèbres de Lie, Chapters IV, V, VI, Hermann, Paris, 1968.

[3] Dynkin, E.B. "Semisimple subalgebras of semisimple Lie algebras." AMS Trnanslations, Series 2, Vol. 6, (1957): 111-244.

[4] Griffiths, D.J., and R. College, Introduction to electrodynamics, Prentice Hall, New Jersey, 1999.

[5] Hakova, L., Hrivnak, J., and J. Patera, "Four families of Weyl group orbit functions of $B_{3}$ and $C_{3}$." J. Math. Phys. 54, 083501 (2013).

[6] Hrivnak, J., and J. Patera, "On discretization of tori of compact simple Lie groups." J. Phys. A: Math. Theor., 42 (2009) 385208; arXiv:0905.2395.

[7] Humphreys, J.E. Introduction to Lie Algebras and Representation Theory, New York, Springer, 1972.

[8] Humphreys, J.E. Reflection Groups and Coxeter Groups, Cambridge Univ. Press, Cambridge, 1990.

[9] Jung:1980, C. "An exactly soluble three-body problem in one-dimension." Can. J. Phys., 58, (1980), 719-728.

[10] Klimyk, A., and J. Patera, "Orbit functions." SIGMA (Symmetry, Integrability and Geometry: Methods and Applications), 2 (2006), 006, 60 pages, math-ph/0601037.

[11] Klimyk A., and J. Patera "Antisymmetric orbit functions." SIGMA, 3 (2007) paper 023, 83 pages, math-ph/0702040v1.

[12] Lemire, F.W., Patera, J., and M. Szajewska, "Dominant weight multiplicities in hybrid characters of $B_{n}, C_{n}, F_{4}, G_{2}$." Internat. J. Theoret. Phys., vol. 54 (11), (2015), 4011-4026.

[13] McKay, W.G., and J. Patera, Tables of dimensions, indices, and branching rules for representations of simple Lie algebras, Marcel Dekker, New York, 1981.
[14] McKay, W.G., Patera, J., and D. Sankoff, "The computation of branching rules for representations of semisimple Lie algebras." Computers in Nonassociative Rings and Algebras, ed. J. Beck and B. Kolman, Academic Press, New York, 1977.

[15] Miller, W. Symmetry and separation of variables, with a foreword by Richard Askey, Encyclopedia of Mathematics and its Applications 4, Addison-Wesley Publishing Co., Reading, Mass.-London-Amsterdam, 1977.

[16] Moody, R.V., Motlochova, L., and J. Patera, "Gaussian cubature arising from hybrid characters of simple Lie groups." J. Fourier Analysis and its Applications, Online ISSN 1531-5851 (2014), 23 pp., arXiv:1202.4415, DOI:10.1007/s00041-014-9355-0

[17] Moody, R.V., and J. Patera, "Characters of elements of finite order in simple Lie groups." SIAM J. on Algebraic and Discrete Methods, 5 (1984), 359-383.

[18] Moon, P., and D.E. Spencer, Field Theory Handbook, Including Coordinate Systems, Differential Equations, and Their Solutions, 2nd ed. New York: Springer-Verlag, 1988.

[19] Nesterenko, M., Patera, J., Szajewska, M., and A. Tereszkiewicz, "Orthogonal polynomials of compact simple Lie groups: Branching rules for polynomials." $J$. Phys. A Math. Theor. 43 (2010), no. 495207, 1-27.

[20] Nesterenko, M., Patera, J., and A. Tereszkiewicz "Orthogonal Polynomials of Compact Simple Lie Groups." Int. J. Math. Math. Sci., (2011), no. 969424, 1-23.

[21] Patera, J."Compact simple Lie groups and theirs $C$-, $S$-, and E-transforms." SIGMA, 1 (2005), 025, 6 pages, math-ph/0512029.

[22] Patera, J., and D. Sankoff, Branching rules for representations of simple Lie algebras, Presses Université de Montréal, Montréal, 1973, 99 pages.

[23] Szajewska, M. "Four types of special functions of $G_{2}$ and their discretization." Integral Transform. Spec. Funct., vol. 23 (6) (2012), 455-472.

[24] Szajewska, M., and A. Tereszkiewicz "Two-dimensional hybrids with mixed boundary value problems." Acta Polytechnica. Journal of advanced engineering, vol. 56 (3) (2016), 245-253.

[25] Tikhonov, A.N., and A.A. Samarskii, Equations of Mathematical Physics, Dover Publ., New York, 1990.

[26] Timoshenko, S., and J.N. Goodier, Theory of Elasticity, McGraw-Hill Book Company, Inc., New York, 1961.

[27] Vinberg, E.B., and A.L. Onishchik Lie groups and Lie algebras, Springer, New York, 1994.

[28] Whippman, M.L. "Branching Rules for Simple Lie Groups." J. Math. Phys., 6 (1965): 1534. 


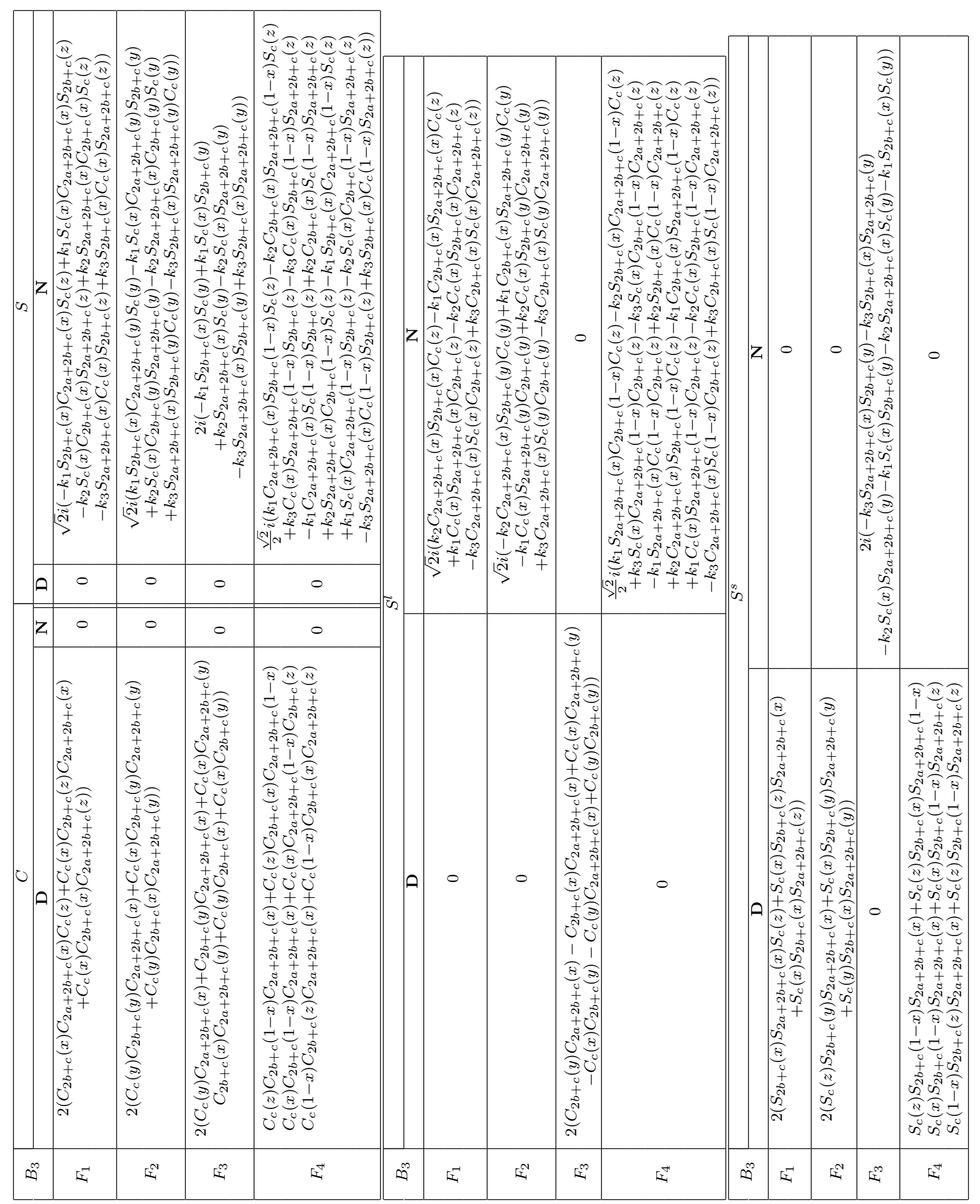

TABle 2. The values of $C$-, $S$-, $S^{l}$ - and $S^{s}$-functions on the boundaries of fundamental region $F$ of $B_{3}$. The separation constants $k_{i}, i=1,2,3$ are given by [6] in $\$ 5.1$ 


\begin{tabular}{|c|c|c|c|c|}
\hline \multirow{2}{*}{$C_{3}$} & \multicolumn{2}{|l|}{$C$} & \multicolumn{2}{|r|}{$S$} \\
\hline & $\mathbf{D}$ & $\mathbf{N}$ & $\mathbf{D}$ & $\mathbf{N}$ \\
\hline$F_{1}$ & $\begin{array}{c}2\left(C_{b+c}(x) C_{c}(y)+C_{a+b+c}(x) C_{c}(y)\right. \\
+C_{a+b+c}(x) C_{b+c}(y)+C_{c}(x) C_{b+c}(y) \\
\left.+C_{c}(x) C_{a+b+c}(y)+C_{b+c}(x) C_{a+b+c}(y)\right) \\
\end{array}$ & 0 & 0 & $\begin{array}{c}-2 i\left(k_{1} S_{b+c}(x) S_{c}(y)+k_{2} S_{a+b+c}(x) S_{c}(y)\right. \\
-k_{3} S_{a+b+c}(x) S_{b+c}(y)+k_{1} S_{c}(x) S_{b+c}(y) \\
-k_{2} S_{c}(x) S_{a+b+c}(y)+k_{3} S_{b+c}(x) S_{a+b+c}(y) \\
\end{array}$ \\
\hline$F_{2}$ & $\begin{array}{l}2\left(C_{a+b+c}(x) C_{b+c}(z) C_{c}(z)\right. \\
+C_{b+c}(x) C_{a+b+c}(z) C_{c}(z) \\
\left.+C_{c}(x) C_{a+b+c}(z) C_{b+c}(z)\right)\end{array}$ & 0 & 0 & $\begin{array}{l}i \sqrt{2}\left(k 1 C_{c}(z) S_{b+c}(x) C_{a+b+c}(z)-k 1 S_{c}(x) S_{b+c}(z) C_{a+b+c}(z)\right. \\
\quad-k 2 S_{c}(z) C_{b+c}(z) S_{a+b+c}(x)+k 2 S_{c}(x) C_{b+c}(z) S_{a+b+c}(z) \\
\left.\quad-k 3 C_{c}(z) S_{b+c}(x) S_{a+b+c}(z)+k 3 C_{c}(z) S_{b+c}(z) S_{a+b+c}(x)\right)\end{array}$ \\
\hline$F_{3}$ & $\begin{array}{l}2\left(C_{c}(z) C_{b+c}(y) C_{a+b+c}(y)\right. \\
+C_{c}(y) C_{b+c}(z) C_{a+b+c}(y) \\
\left.+C_{c}(y) C_{b+c}(y) C_{a+b+c}(z)\right)\end{array}$ & 0 & 0 & $\begin{array}{c}-i \sqrt{2}\left(k 1 S_{c}(z) S_{b+c}(y) C_{a+b+c}(y)-k 1 S_{c}(y) S_{b+c}(z) C_{a+b+c}(y)\right. \\
-k 2 S c(z) C_{b+c}(y) S_{a+b+c}(y)+k 2 S_{c}(y) C_{b+c}(y) S_{a+b+c}(z) \\
\left.-k 3 C_{c}(y) S_{b+c}(y) S_{a+b+c}(z)+k 3 C_{c}(y) S_{b+c}(z) S a+b+c(y)\right)\end{array}$ \\
\hline$F_{4}$ & $\begin{array}{l}C_{a+b+c}\left(\frac{1}{\sqrt{2}}\right) C_{b+c}(y) C_{c}(z) \\
+C_{b+c}\left(\frac{1}{\sqrt{2}}\right) C_{a+b+c}(y) C_{c}(z) \\
+C_{c}\left(\frac{1}{\sqrt{2}}\right) C_{a+b+c}(y) C_{b+c}(z) \\
\end{array}$ & 0 & 0 & $\begin{array}{r}i\left(k 1 S_{c}(z) C_{a+b+c}\left(\frac{1}{\sqrt{2}}\right) S_{b+c}(y)-k 1 S_{c}(y) C_{a+b+c}\left(\frac{1}{\sqrt{2}}\right) S_{b+c}(z)\right. \\
-k 2 C_{b+c}\left(\frac{1}{\sqrt{2}}\right) S_{c}(z) S_{a+b+c}(y)+k 2 C_{b+c}\left(\frac{1}{\sqrt{2}}\right) S_{c}(y) S_{a+b+c}(z) \\
\left.-k 3 C_{c}\left(\frac{1}{\sqrt{2}}\right) S_{b+c}(y) S_{a+b+c}(z)+k 3 C_{c}\left(\frac{1}{\sqrt{2}}\right) S_{b+c}(z) S_{a+b+c}(y)\right) \\
\end{array}$ \\
\hline \multirow{2}{*}{$C_{3}$} & \multicolumn{4}{|r|}{$\begin{array}{ll}S^{l} \\
\end{array}$} \\
\hline & \multicolumn{3}{|l|}{ D } & $\mathbf{N}$ \\
\hline$F_{1}$ & \multicolumn{3}{|l|}{0} & $\begin{aligned} & -2 i\left(k_{1} S_{b+c}(x) C_{c}(y)+k_{2} S_{a+b+c}(x) S_{c}(y)\right. \\
& +k_{3} S_{a+b+c}(x) S_{b+c}(y)+k_{1} S_{c}(x) S_{b+c}(y) \\
+ & \left.k_{2} S_{c}(x) S_{a+b+c}(y)+k_{3} S_{b+c}(x) S_{a+b+c}(y)\right)\end{aligned}$ \\
\hline$F_{2}$ & \multicolumn{3}{|c|}{$\begin{array}{c}2\left(S_{a+b+c}(x) S_{b+c}(z) S_{c}(z)+S_{b+c}(x) S_{a+b+c}(z) S_{c}(z)\right. \\
\left.+S_{c}(x) S_{a+b+c}(z) S_{b+c}(z)\right)\end{array}$} & 0 \\
\hline$F_{3}$ & \multicolumn{3}{|c|}{$\begin{array}{c}2\left(S_{c}(z) S_{b+c}(y) S_{a+b+c}(y)+S_{c}(y) S_{b+c}(z) S_{a+b+c}(y)\right. \\
\left.+S_{c}(y) S_{b+c}(y) S_{a+b+c}(z)\right)\end{array}$} & 0 \\
\hline$F_{4}$ & \multicolumn{3}{|l|}{0} & $\begin{array}{l}i\left(k 1 S_{c}(z) C_{a+b+c}\left(\frac{1}{\sqrt{2}}\right) S_{b+c}(y)+k 1 S_{c}(y) C_{a+b+c}\left(\frac{1}{\sqrt{2}}\right) S_{b+c}(z)\right. \\
+k 2 C_{b+c}\left(\frac{1}{\sqrt{2}}\right) S_{c}(z) S_{a+b+c}(y)+k 2 C_{b+c}\left(\frac{1}{\sqrt{2}}\right) S_{c}(y) S_{a+b+c}(z) \\
\left.+k 3 C_{c}\left(\frac{1}{\sqrt{2}}\right) S_{b+c}(y) S_{a+b+c}(z)+k 3 C_{c}\left(\frac{1}{\sqrt{2}}\right) S_{b+c}(z) S_{a+b+c}(y)\right) \\
\end{array}$ \\
\hline \multirow{2}{*}{$C_{3}$} & \multicolumn{4}{|r|}{$\bar{~} \overline{S^{S}}$} \\
\hline & \multicolumn{3}{|c|}{$\begin{array}{c}\text { D } \\
-2\left(C_{b+c}(x) C_{a+b+c}(y)-C_{c}(y) C_{a+b+c}(x)\right.\end{array}$} & $\mathbf{N}$ \\
\hline$F_{1}$ & \multicolumn{3}{|c|}{$\begin{array}{l}-2\left(C_{b+c}(x) C_{a+b+c}(y)-C_{c}(y) C_{a+b+c}(x)\right. \\
+C_{b+c}(y) C_{a+b+c}(x)+C_{c}(x) C_{a+b+c}(y) \\
\left.\quad+C_{c}(y) C_{b+c}(x)-C_{c}(x) C_{b+c}(y)\right)\end{array}$} & 0 \\
\hline$F_{2}$ & \multicolumn{3}{|l|}{0} & $\begin{array}{l}\sqrt{2}\left(k 1 C_{c}(z) C_{b+c}(x) S_{a+b+c}(z)-k 1 C_{c}(x) C_{b+c}(z) S_{a+b+c}(z)\right. \\
\quad-k 2 C_{c}(z) S_{b+c}(z) C_{a+b+c}(x)+k 2 C_{c}(x) S_{b+c}(z) C_{a+b+c}(z) \\
\left.+k 3 S_{c}(z) C_{b+c}(z) C_{a+b+c}(x)-k 3 S_{c}(z) C_{b+c}(x) C_{a+b+c}(z)\right) \\
\end{array}$ \\
\hline$F_{3}$ & \multicolumn{3}{|l|}{0} & $\begin{array}{l}-i \sqrt{2}\left(k 1 C_{c}(z) C_{b+c}(y) S_{a+b+c}(y)-k 1 C_{c}(y) C_{b+c}(z) S_{a+b+c}(y)\right. \\
\quad-k 2 C_{c}(z) S_{b+c}(y) C_{a+b+c}(y)+k 2 C_{c}(y) S_{b+c}(y) C_{a+b+c}(z) \\
\left.\quad+k 3 S_{c}(y) C_{b+c}(z) C_{a+b+c}(y)-k 3 S_{c}(y) C_{b+c}(y) C_{a+b+c}(z)\right) \\
\end{array}$ \\
\hline$F_{4}$ & \multicolumn{3}{|c|}{$\begin{array}{l}C_{c}(z) C_{a+b+c}\left(\frac{1}{\sqrt{2}}\right) C_{b+c}(y)-C_{c}(y) C_{a+b+c}\left(\frac{1}{\sqrt{2}}\right) C_{b+c}(z) \\
-C_{b+c}\left(\frac{1}{\sqrt{2}}\right) C_{c}(z) C_{a+b+c}(y)+C_{c}\left(\frac{1}{\sqrt{2}}\right) C_{b+c}(z) C_{a+b+c}(y) \\
+C_{b+c}\left(\frac{1}{\sqrt{2}}\right) C_{c}(y) C_{a+b+c}(z)-C_{c}\left(\frac{1}{\sqrt{2}}\right) C_{b+c}(y) C_{a+b+c}(z)\end{array}$} & 0 \\
\hline
\end{tabular}

TABle 3. The values of $C_{-}, S_{-}, S^{l}$ - and $S^{s}$-functions on the boundaries of fundamental region $F$ of $C_{3}$. The separation constants $k_{i}, i=1,2,3$ are given by 7 in $\$ 5.1$ 


\begin{tabular}{|c|c|c|c|c|c|c|c|c|}
\hline \multirow{2}{*}{$C_{2} \times A_{1}$} & \multicolumn{5}{|c|}{$C$} & \\
\hline & \multicolumn{4}{|c|}{$\mathbf{D}$} & $\mathbf{N}$ & \multicolumn{3}{|c|}{$\frac{S}{\mathbf{N}}$} \\
\hline$F_{1}$ & \multicolumn{4}{|c|}{$2\left(C_{a+b}(x) C_{b}(y)+C_{b}(x) C_{a+b}(y)\right)$} & 0 & 0 & $-2 i k_{3}\left(S_{a+b}(x) S_{b}(y)-S_{b}(x\right.$ & $\left.S_{a+b}(y)\right)$ \\
\hline$F_{2}$ & \multicolumn{4}{|c|}{$2\left(C_{a+b}(x) C_{c}(z)+C_{b}(x) C_{c}(z)\right)$} & 0 & 0 & $-2 i k_{2} S_{a+b}(x) S_{c}(z)+i k_{1} S$ & $(x) S_{c}(z)$ \\
\hline$F_{3}$ & \multicolumn{4}{|c|}{$2 C_{a+b}(y) C_{b}(y) C_{c}(z)$} & 0 & 0 & $-i \sqrt{2}\left(k_{1} C_{a+b}(y) S_{b}(y) S_{c}(z)-k_{2} C_{t}\right.$ & $(y) S_{a+b}(y) S_{c}(z)$ \\
\hline$F_{4}$ & \multicolumn{4}{|c|}{$C_{a+b}\left(\frac{\sqrt{2}}{2}\right) C_{b}(y) C_{c}(z)+C_{b}\left(\frac{\sqrt{2}}{2}\right) C_{a+b}(y) C_{c}(z)$} & 0 & 0 & $i k_{1} C_{a+b}\left(\frac{\sqrt{2}}{2}\right) S_{b}(y) S_{c}(z)-i k_{2} C_{b}($ & $\left.\sqrt{\frac{2}{2}}\right) S_{a+b}(y) S_{c}(z)$ \\
\hline$F_{5}$ & \multicolumn{4}{|c|}{$C_{a+b}(x) C_{b}(y) C_{c}\left(\frac{\sqrt{2}}{2}\right)+C_{b}(x) C_{a+b}(y) C_{c}\left(\frac{\sqrt{2}}{2}\right)$} & 0 & 0 & $i k_{3}\left(S_{a+b}(x) S_{b}(y) C_{c}\left(\frac{\sqrt{2}}{2}\right)-S_{b}(x)\right.$ & $\left.S_{a+b}(y) C_{c}\left(\frac{\sqrt{2}}{2}\right)\right)$ \\
\hline \multirow{7}{*}{55} & & \multirow{2}{*}{$C_{2} \times A_{1}$} & & \\
\hline & & & \multicolumn{4}{|l|}{$\mathbf{D}$} & $\mathbf{N}$ & \\
\hline & & $F_{1}$ & 0 & \multicolumn{4}{|c|}{$-2 i k_{3}\left(S_{a+b}(x) S_{b}(y)+S_{b}(x) S_{a+b}(y)\right)$} & \\
\hline & & $F_{2}$ & 0 & \multicolumn{4}{|c|}{$-2 i\left(k_{2} S_{a+b}(x) S_{c}(z)+k_{1} S_{b}(x) S_{c}(z)\right)$} & \\
\hline & & $F_{3}$ & $2 S_{a+b}(y) S_{b}(y) S_{c}(z)$ & & & & 0 & \\
\hline & & $F_{4}$ & 0 & $i\left(k_{1}\right.$ & $a+b$ & $\left.\frac{\sqrt{2}}{2}\right) S$ & $\left.(y) S_{c}(z)+k_{2} C_{b}\left(\frac{\sqrt{2}}{2}\right) S_{a+b}(y) S_{c}(z)\right)$ & \\
\hline & & $F_{5}$ & 0 & $i k_{3}$ & $S_{a+b}$ & $x) S_{b}$ & $\left.y) C_{c}\left(\frac{\sqrt{2}}{2}\right)+S_{b}(x) S_{a+b}(y) C_{c}\left(\frac{\sqrt{2}}{2}\right)\right)$ & \\
\hline \multirow{2}{*}{\multicolumn{2}{|c|}{$C_{2} \times A_{1}$}} & \multicolumn{7}{|c|}{$\overline{S^{s}}$} \\
\hline & & \multicolumn{2}{|r|}{$\mathbf{D}$} & & & \multicolumn{3}{|c|}{$\mathbf{N}$} \\
\hline \multicolumn{2}{|c|}{$F_{1}$} & \multicolumn{4}{|c|}{$2\left(C_{a+b}(x) C_{b}(y)-C_{b}(x) C_{a+b}(y)\right)$} & \multicolumn{3}{|c|}{0} \\
\hline \multicolumn{2}{|c|}{$F_{2}$} & \multicolumn{4}{|c|}{$2\left(C_{a+b}(x) C_{c}(z)-C_{b}(x) C_{c}(z)\right)$} & \multicolumn{3}{|c|}{0} \\
\hline \multicolumn{2}{|c|}{$F_{3}$} & \multicolumn{4}{|c|}{0} & \multicolumn{3}{|c|}{$-i \sqrt{2}\left(k_{1} S_{a+b}(y) C_{b}(y) C_{c}(z)-k_{2} S_{b}(y) C_{a+b}(y) C_{c}(z)\right)$} \\
\hline$F$ & & $C_{a+b}\left(\frac{\sqrt{2}}{2}\right) C$ & $(y) C_{c}(z)-C_{b}\left(\frac{\sqrt{2}}{2}\right) C_{a \dashv}$ & ${ }_{b}(y) C$ & & & 0 & \\
\hline$F$ & & $C_{a+b}(x) C_{b}$ & $C_{c}\left(\frac{\sqrt{2}}{2}\right)-C_{b}(x) C_{a+b}$ & $y) C_{c}($ & & & 0 & \\
\hline
\end{tabular}

TABLE 4. The values of $C$-, $S$-, $S^{l}$ - and $S^{s}$-functions on the boundaries of fundamental region $F$ of $C_{2} \times A_{1}$. The separation constants $k_{i}, i=1,2,3$ are given by 8 in $\S 5.2$ 


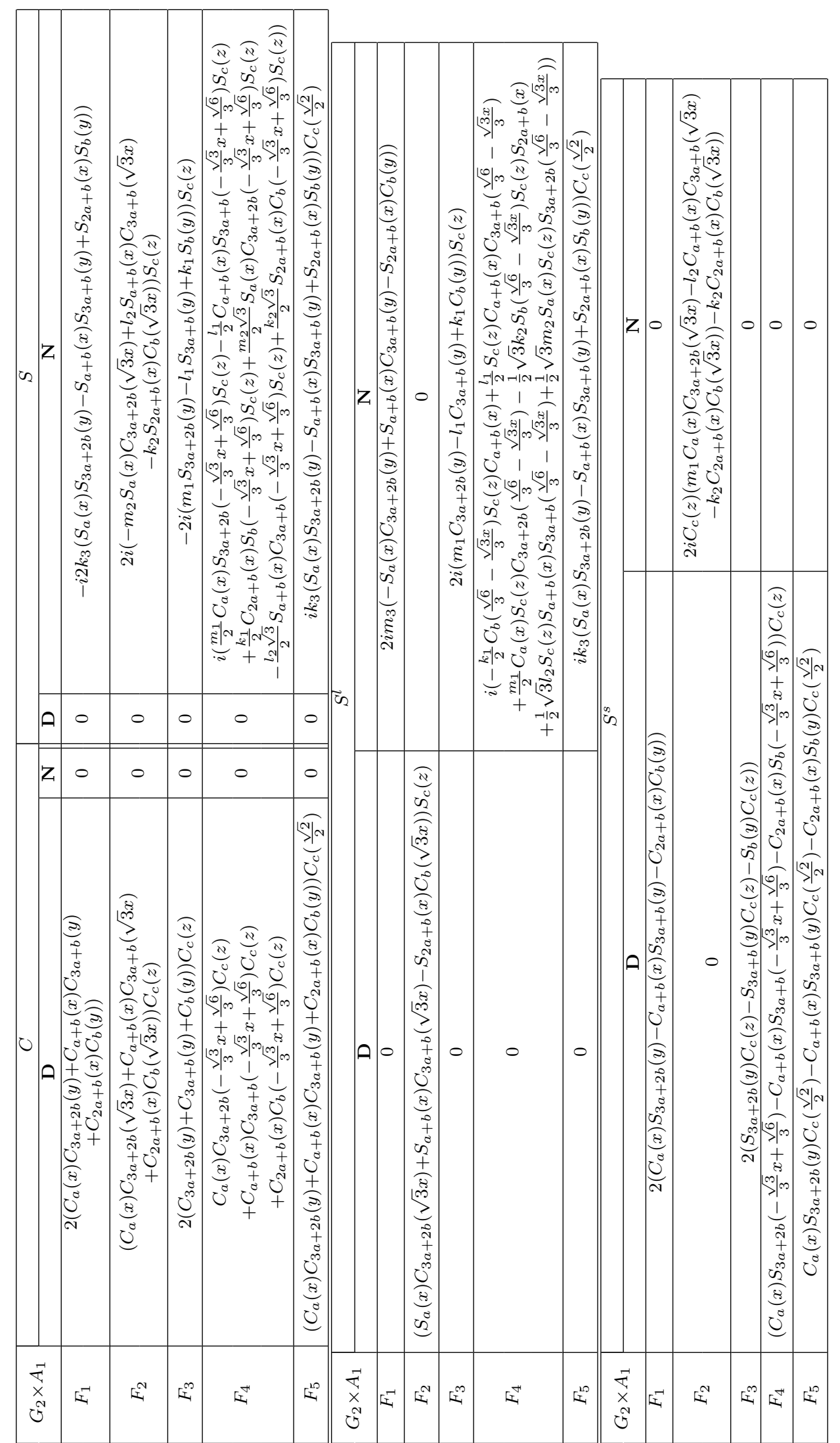

TABle 5. The values of $C$-, $S$-, $S^{l}$ - and $S^{s}$-functions on the boundaries of fundamental region $F$ of $G_{2} \times A_{1}$. The separation constants $k_{i}, l_{i}, m_{i}, i=1,2,3$ are given by 9 in $\$ 5.2$ 


\begin{tabular}{|c|c|c|c|c|c|c|c|}
\hline & \multirow{3}{*}{$A_{1} \times A_{1} \times A_{1}$} & & & & & & \\
\hline & & \multicolumn{2}{|l|}{$C C C$} & \multicolumn{3}{|c|}{$S S S$} & \\
\hline & & $\mathbf{D}$ & $\mathbf{N}$ & \multicolumn{3}{|c|}{$\frac{S S S}{\mathbf{N}}$} & \\
\hline & $F_{1}$ & \multirow{2}{*}{$\begin{array}{l}C_{a}(x) C_{b}(y) C_{c}(0) \\
C_{a}(x) C_{b}(0) C_{c}(z)\end{array}$} & 0 & 0 & \multicolumn{2}{|c|}{$-\sqrt{2} \pi i k_{3} S_{a}(x) S_{b}(y) C_{c}(0)$} & \\
\hline & $F_{2}$ & & 0 & 0 & \multicolumn{2}{|c|}{$-\sqrt{2} \pi i k_{2} S_{a}(x) C_{b}(0) S_{c}(z)$} & \\
\hline & $F_{3}$ & $C_{a}(0) C_{b}(y) C_{c}(z)$ & 0 & 0 & \multicolumn{2}{|c|}{$-\sqrt{2} \pi i k_{1} C_{a}(0) S_{b}(y) S_{c}(z)$} & \\
\hline & $F_{4}$ & $C_{a}(x) C_{b}\left(\frac{1}{\sqrt{2}}\right) C_{c}(z)$ & 0 & 0 & \multicolumn{2}{|c|}{$\sqrt{2} \pi i k_{2} S_{a}(x) C_{b}\left(\frac{1}{\sqrt{2}}\right) S_{c}(z)$} & \\
\hline & $F_{5}$ & $C_{a}\left(\frac{1}{\sqrt{2}}\right) C_{b}(y) C_{c}(z)$ & 0 & 0 & \multicolumn{2}{|c|}{$\sqrt{2} \pi i k_{1} C_{a}\left(\frac{1}{\sqrt{2}}\right) S_{b}(y) S_{c}(z)$} & \\
\hline & $F_{6}$ & $C_{a}(x) C_{b}(y) C_{c}\left(\frac{1}{\sqrt{2}}\right)$ & 0 & \multirow[t]{2}{*}{0} & \multicolumn{2}{|c|}{$\sqrt{2} \pi i k_{3} S_{a}(x) S_{b}(y) C_{c}\left(\frac{1}{\sqrt{2}}\right)$} & \\
\hline \multirow{2}{*}{$A_{1} \times A_{1} \times A_{1}$} & \multicolumn{3}{|c|}{$C C S$} & & \multicolumn{3}{|c|}{$S S C$} \\
\hline & $\mathbf{D}$ & \multicolumn{2}{|l|}{$\mathbf{N}$} & & $\mathbf{D}$ & & $\mathbf{N}$ \\
\hline$F_{1}$ & 0 & \multicolumn{3}{|c|}{$-\sqrt{2} \pi i k_{3} C_{a}(x) C_{b}(y) C_{c}(0)$} & $S_{a}(x) S_{b}(y) C_{c}(0)$ & & 0 \\
\hline$F_{2}$ & $C_{a}(x) C_{b}(0) S_{c}(z)$ & \multicolumn{3}{|c|}{0} & 0 & $-\sqrt{2} \pi i$ & ${ }_{2} S_{a}(x) C_{b}(0) C_{c}(z)$ \\
\hline$F_{3}$ & $C_{a}(0) C_{b}(y) S_{c}(z)$ & 0 & & & 0 & $-\sqrt{2} \pi i$ & ${ }_{1} C_{a}(0) S_{b}(y) C_{c}(z)$ \\
\hline$F_{4}$ & $C_{a}(x) C_{b}\left(\frac{1}{\sqrt{2}}\right) S_{c}(z)$ & 0 & & & 0 & $\sqrt{2} \pi i k_{2}$ & ${ }_{a}(x) C_{b}\left(\frac{1}{\sqrt{2}}\right) C_{c}(z)$ \\
\hline$F_{5}$ & $C_{a}\left(\frac{1}{\sqrt{2}}\right) C_{b}(y) S_{c}(z)$ & 0 & & & 0 & $\sqrt{2} \pi i k_{1}$ & $C_{a}\left(\frac{1}{\sqrt{2}}\right) S_{b}(y) C_{c}(z)$ \\
\hline$F_{6}$ & 0 & $\sqrt{2} \pi i k_{3} C_{a}(x) C_{b}(y)$ & $c\left(\frac{1}{\sqrt{2}}\right)$ & & $S_{a}(x) S_{b}(y) C_{c}\left(\frac{1}{\sqrt{2}}\right)$ & & 0 \\
\hline & & $C S C$ & & & & $S C S$ & \\
\hline$A_{1} \times A_{1} \times A_{1}$ & $\mathbf{D}$ & $\mathbf{N}$ & & & $\mathbf{D}$ & & $\mathbf{N}$ \\
\hline$F_{1}$ & $C_{a}(x) S_{b}(y) C_{c}(0)$ & 0 & & & 0 & $-\sqrt{2} \pi i$ & ${ }_{3} S_{a}(x) C_{b}(y) C_{c}(0)$ \\
\hline$F_{2}$ & 0 & $-\sqrt{2} \pi i k_{2} C_{a}(x) C_{b}($ & $C_{c}(z)$ & & $S_{a}(x) C_{b}(0) S_{c}(z)$ & & 0 \\
\hline$F_{3}$ & $C_{a}(0) S_{b}(y) C_{c}(z)$ & 0 & & & 0 & $-\sqrt{2} \pi i$ & ${ }_{1} C_{a}(0) C_{b}(y) S_{c}(z)$ \\
\hline$F_{4}$ & 0 & $\sqrt{2} \pi i k_{2} C_{a}(x) C_{b}\left(\frac{1}{\sqrt{ }}\right.$ & $C_{c}(z)$ & & $S_{a}(x) C_{b}\left(\frac{1}{\sqrt{2}}\right) S_{c}(z)$ & & 0 \\
\hline$F_{5}$ & $C_{a}\left(\frac{1}{\sqrt{2}}\right) S_{b}(y) C_{c}(z)$ & 0 & & & 0 & $\sqrt{2} \pi i k_{1}$ & $C_{a}\left(\frac{1}{\sqrt{2}}\right) C_{b}(y) S_{c}(z)$ \\
\hline$F_{6}$ & $C_{a}(x) S_{b}(y) C_{c}\left(\frac{1}{\sqrt{2}}\right)$ & 0 & & & 0 & $\sqrt{2} \pi i k_{3}$ & ${ }_{a}(x) C_{b}(y) C_{c}\left(\frac{1}{\sqrt{2}}\right)$ \\
\hline & & $S C C$ & & & & $\overline{C S S}$ & \\
\hline$A_{1} \times A_{1} \times A_{1}$ & $\mathbf{D}$ & $\mathbf{N}$ & & & $\mathbf{D}$ & & $\mathbf{N}$ \\
\hline$F_{1}$ & $S_{a}(x) C_{b}(y) C_{c}(0)$ & 0 & & & 0 & $-\sqrt{2} \pi i$ & ${ }_{3} C_{a}(x) S_{b}(y) C_{c}(0)$ \\
\hline$F_{2}$ & $S_{a}(x) C_{b}(0) C_{c}(z)$ & 0 & & & 0 & $-\sqrt{2} \pi i$ & ${ }_{2} C_{a}(x) C_{b}(0) S_{c}(z)$ \\
\hline$F_{3}$ & 0 & $-\sqrt{2} \pi i k_{1} C_{a}(0) C_{b}$ & $C_{c}(z)$ & & $C_{a}(0) S_{b}(y) S_{c}(z)$ & & 0 \\
\hline$F_{4}$ & $S_{a}(x) C_{b}\left(\frac{1}{\sqrt{2}}\right) C_{c}(z)$ & 0 & & & 0 & $\sqrt{2} \pi i k_{2}$ & $C_{a}(x) C_{b}\left(\frac{1}{\sqrt{2}}\right) S_{c}(z)$ \\
\hline$F_{5}$ & 0 & $\sqrt{2} \pi i k_{1} C_{a}\left(\frac{1}{\sqrt{2}}\right) C_{b}$ & ${ }_{1} C_{c}(z$ & & $C_{a}\left(\frac{1}{\sqrt{2}}\right) S_{b}(y) S_{c}(z)$ & & 0 \\
\hline$F_{6}$ & $S_{a}(x) C_{b}(y) C_{c}\left(\frac{1}{\sqrt{2}}\right)$ & 0 & & & 0 & $\sqrt{2} \pi i k_{3}$ & $C_{a}(x) S_{b}(y) C_{c}\left(\frac{1}{\sqrt{2}}\right)$ \\
\hline
\end{tabular}

TABLE 6 . The values of six families of functions on the boundaries of fundamental region $F$ of $A_{1} \times A_{1} \times A_{1}$. The separation constants $k_{i}, i=1,2,3$ are given by 10 in $\S 5.3$ 